\title{
Diverse protist grazers select for virulence-related traits in Legionella
}

\author{
Francisco Amaro ${ }^{1}$, Wen Wang ${ }^{2}$, Jack A Gilbert ${ }^{3}$, O Roger Anderson ${ }^{4}$ and Howard A Shuman ${ }^{1}$ \\ ${ }^{1}$ Department of Microbiology, University of Chicago, Chicago, IL, USA; ${ }^{2} J i a n g s u$ Key Laboratory for \\ Biodiversity \& Biotechnology, Nanjing Normal University, Nanjing, People's Republic of China; ${ }^{3}$ Biology \\ Division, Argonne National Laboratory and Department of Ecology \& Evolution, University of Chicago, \\ Chicago, IL, USA and ${ }^{4}$ Division of Biology and Paleo Environment, Lamont-Doherty Earth Observatory, \\ Columbia University, Palisades, NY, USA
}

\begin{abstract}
It is generally accepted that selection for resistance to grazing by protists has contributed to the evolution of Legionella pneumophila as a pathogen. Grazing resistance is becoming more generally recognized as having an important role in the ecology and evolution of bacterial pathogenesis. However, selection for grazing resistance presupposes the existence of protist grazers that provide the selective pressure. To determine whether there are protists that graze on pathogenic Legionella species, we investigated the existence of such organisms in a variety of environmental samples. We isolated and characterized diverse protists that graze on $L$. pneumophila and determined the effects of adding $L$. pneumophila on the protist community structures in microcosms made from these environmental samples. Several unrelated organisms were able to graze efficiently on L. pneumophila. The community structures of all samples were markedly altered by the addition of L. pneumophila. Surprisingly, some of the Legionella grazers were closely related to species that are known hosts for $L$. pneumophila, indicating the presence of unknown specificity determinants for this interaction. These results provide the first direct support for the hypothesis that protist grazers exert selective pressure on Legionella to acquire and retain adaptations that contribute to survival, and that these properties are relevant to the ability of the bacteria to cause disease in people. We also report a novel mechanism of killing of amoebae by one Legionella species that requires an intact Type IV secretion system but does not involve intracellular replication. We refer to this phenomenon as 'food poisoning'.

The ISME Journal (2015) 9, 1607-1618; doi:10.1038/ismej.2014.248; published online 9 January 2015
\end{abstract}

\section{Introduction}

The interaction of bacteria and protists is considered to be one of the oldest prey-predator interactions in nature (Cavalier-Smith, 2002). In aquatic environments, soil or anthropogenic ecosystems, predation by phagocytic protists is a major cause of bacterial mortality and a significant driving force of the genetic and functional structure of bacterial communities (Pernthaler, 2005: Bell et al., 2010: Jousset, 2012). Because bacteria that resist grazing by protists will show increased environmental fitness (Hahn and Hofle, 2001), a variety of anti-predator strategies, such as morphological adaptations, production of toxic secondary metabolites or resistance to digestion, have evolved (Matz and Kjelleberg, 2005; Jousset, 2012). In addition to acting as predators, free-living

Correspondence: H Shuman, Department of Microbiology, University of Chicago, 920 East 58th Street, Chicago, IL 60637, USA. E-mail: hashuman@uchicago.edu

Received 2 June 2014; revised 12 November 2014; accepted 19 November 2014; published online 9 January 2015 protists can also provide bacteria a protective niche and source of nutrients to be exploited. Thus, bacterial traits that may have originally evolved to survive phagocytosis can also be used to exploit protists as hosts, and might facilitate the transition from a grazing-resistant bacterium to an endosymbiont or intracellular pathogen (Matz and Kjelleberg, 2005). The human pathogen Legionella pneumophila represents a well-known example of a grazing-resistant bacterial species that can also take advantage of protists as a protective niche (Kilvington and Price, 1990).

L. pneumophila is a gram-negative $\gamma$-proteobacterial species common in soil and natural or man-made freshwater sources, where it survives by growing inside different species of free-living protists (Rowbotham, 1980; Fields, 1996). L. pneumophila can cause an acute, life-threatening pneumonia called Legionnaires' disease (McDade et al., 1977) and less severe, self-limiting disease called Pontiac Fever (Fraser et al., 1977). Humans become infected by inhalation of contaminated aerosols produced in man-made water systems. Once inside the human lung, L. pneumophila infects and replicates within 
alveolar macrophages in a process similar to the one that occurs within amoebae. (Nash et al., 1984, 1988; Al-Quadan et al., 2012).

The ability of Legionella to replicate inside protists and macrophages requires a complex Type IV-B secretion system (TFBSS) called the Icm/Dot system (Segal et al., 1998; Vogel et al., 1998), which delivers a repertoire of $\sim 300$ effector proteins to host cells (Berger and Isberg, 1993; Segal and Shuman, 1999; Gomez-Valero et al., 2011), where they manipulate a variety of host cell processes. The Dot/Icm system is therefore the major virulence determinant for this pathogen. The secreted effector proteins prevent fusion of the Legionella-containing vacuole with lysosomes and promote its development into a replication compartment (Ninio and Roy, 2007; Franco et al., 2009; Luo, 2012; Segal, 2013). Curiously, nearly all identified effector proteins appear to be functionally redundant in that null mutations that eliminate single or even multiple effectors result in no obvious decrease in the ability to cause infection or survive grazing (Ninio and Roy, 2007; Ensminger and Isberg, 2009; O’Connor et al., 2011).

One possible explanation for the origin of effector redundancy is that selection for survival and replication in a variety of environmental hosts has resulted in the acquisition of a pool of genes, encoding many effectors with overlapping functions and/or specificities. Indeed, L. pneumophila replicates within and kills many phylogenetically diverse water and soil protists (Rowbotham, 1980; Fields, 1996; Solomon et al., 2000; Molmeret et al., 2005). The TFBSS is also absolutely required for survival and growth in the protozoan hosts and there are no major differences in the functional requirements for Legionella replication in amoebae and macrophages (Gao et al., 1997; Segal and Shuman, 1999). Therefore, it has been proposed that the ability of $L$. pneumophila to parasitize human macrophages is a consequence of is prior adaptation to intracellular growth in protists (AlQuadan et al., 2012). Predation by protozoa has been proposed to provide the selective pressure for acquisition and maintenance of properties that contribute to virulence, but there is no experimental evidence of protozoa grazing on Legionella.

In this study, we investigated the existence of protozoa from different environments that are able to ingest and kill Legionella. By performing microcosm experiments and $18 \mathrm{~S}$ small subunit (SSU) pyrosequencing, we identified different unrelated protozoan morphotypes that consume Legionella as a source of food and have studied the impact of $L$. pneumophila on the structure of eukaryotic microbial communities. These studies provide clear evidence for the prevalence of predators that exert selective pressure on $L$. pneumophila to resist grazing. In addition, we were able to cultivate representative species of these organisms and study their interactions with L. pneumophila.

\section{Materials and methods}

\section{Microcosm design}

Environmental samples (soil, fresh water and ocean sediment) used in this study are listed in Supplementary Table S3. Microcosms were set up in sterile polystyrene Petri dishes containing nonnutrient agar. Each soil sample $(\sim 1.5 \mathrm{~g})$ was suspended in $1.5 \mathrm{ml}$ of $0.2 \mu \mathrm{m}$ micropore-filtered pond water. On a glass microfiber filter on the surface of the agar, $0.5 \mathrm{~g}$ of this mixture was deposited. The agar was covered with $10 \mathrm{ml}$ of sterile pond water and three cubes $\left(1 \mathrm{~cm}^{2}\right)$ of malt yeast extract agar (Page, 1988) were added to promote growth of native bacteria. Three plates were set up for each environmental sample. After 10 days of incubation at room temperature in the dark, the microbial community was harvested from one plate (plate $t=0$ : this one would be considered as the initial population in the microcosm) and RNA was extracted with Trizol reagent (Invitrogen, Grand Island, NY, USA). The two remaining plates were inoculated with $10^{7}$ cells of either Escherichia coli (plate EC) or L. pneumophila strain Philadelphia-1 (plate Phi) and incubated for 10 days at room temperature. After that, RNA from each plate was extracted and stored at $-80^{\circ} \mathrm{C}$. For fresh-water samples, 1-2 liter of water was filtered through a $0.25 \mu \mathrm{m}$ micropore filter. The filter was deposited on the surface of non-nutrient agar plates and microcosms were set up, as described above. Details on the reverse transcription PCR amplification and pyrosequencing protocol are provided as Supplementary Material.

Changes in the microbial community in microcosms can also occur during incubation owing to the addition of a bacterial potential prey, possibly providing added nourishment for some protists. To circumvent this limitation, we performed control microcosms, in which each environmental sample was incubated with $E$. coli as prey instead of $L$. pneumophila. Encystment of free-living amoebae occurs under various conditions, such as nutrient starvation and response to bacterial toxins (Fouque et al., 2012). Thus, microcosms without any bacteria added would result in encystment of the starving protists. As the cell envelope of L. pneumophila displays virulence properties (Shevchuk et al., 2011), we believe that the addition of E. coli represents a more suitable control rather than the Type IV secretion-deficient $\operatorname{dot} A$ mutant strain of $L$. pneumophila.

\section{Sequence data analyses and taxonomic affiliation}

Sequence data were processed for quality and analyzed using the QIIME 1.6.0 software pipeline as described in Caporaso et al. (2011). Reads were truncated at their first low-quality base. Then, reads shorter than 75 bases were discarded, as were reads whose barcode did not match an expected barcode. Chimera checking and operational taxonomic units (OTUs) grouping were performed in QIIME using 
Usearch (Edgar et al., 2011). Taxonomic assignment of recovered eukaryotic OTUs (determined at $\geqslant 97 \%$ similarity) by basic local alignment search tool, nucleotide searches against the SILVA comprehensive ribosomal RNA database (Quast et al., 2013). After taxonomic affiliation was assigned, a data set comprising only high-quality protist sequences (4 225703 sequences from the 42 microcosms examined, Supplementary Table S3) was culled from all high-quality sequence reads. Further analyses were carried out on the protist data set rarefied to 11203 sequences to correct for uneven sampling efforts. The processed DNA sequences obtained in this study have been deposited in the NCBI Sequence Read Archive database under the accession number SUB472144.

Isolation of protists resistant to L. pneumophila Protist morphotypes were subcultured from microcosms incubated with $L$. pneumophila by transferring them to new agar plate covered with sterile pond water and Legionella as a food source. Clones of each protist morphotype were isolated using a sterile agar separation technique (Page, 1988). Protists were suspended in sterile pond water and small droplets of the suspension were deposited on the surface of sterile non-nutrient agar plates. On successive days, plates were examined to detect any protists that had migrated out of the droplets onto the sterile surface. Isolated protists were picked from the surface of the agar with a sterile spatula and deposited in new agar plates. Subcultures of the clones were maintained by routinely transferring to a new culture media, consisting on pond water and E. coli as prey. A sequence of $18 \mathrm{~S}$ rDNA of particular protist isolates (CWPL, MG33, C37C6, D5MG, WPD37) was amplified from RNA by reverse transcription PCR with the Euk1A and Euk516R primers (Supplementary Table S2). Reverse transcription PCR products were cloned into the pGEMT-easy vector (Promega, Madison, WI, USA). Selected clones were sequenced in both directions using the universal M13-promoter primer. Taxonomic affiliation of each isolate was assigned by phylogenetic analysis and comparison with $18 \mathrm{~S}$ SSU sequences in the NCBI nr database.

Co-cultivation of protists with different Legionella strains and quantification of protozoan growth and Legionella consumption by real-time PCR assays

Legionella strains were tested by co-cultivation with each protist isolate in 48-well plates. Each well containing $0.5 \mathrm{ml}$ of sterile filtered pond water was seeded with $2 \times 10^{4}$ trophozoites. Legionella strains grown on charcoal yeast extract medium were suspended in sterile pond water and inoculated at the desired multiplicity of infection (MOI: ratio of Legionella cells added per protist cell). Wells were monitored for protist growth or death and bacterial consumption for up to 10 days at room temperature.
Protozoan growth and Legionella consumption was measured by real-time PCR assays. Genomic DNA was extracted using the AxyPrep Blood Genomic DNA Miniprep kit (Axygen, Union City, CA, USA) from protist-Legionella co-cultures. Genome equivalents (GE) for each protist morphotype and Legionella strain were quantified using the primers that are listed in Supplementary Table S2. Targets for real-time PCR were the genes coding for the Solumitrus palustris $18 \mathrm{~S}$ rDNA, Paracercomonas CWPL $18 \mathrm{~S}$ rDNA, Cercomonas MG33, L. pneumophila $23 \mathrm{~S}-5 \mathrm{~S}$ rDNA internal transcribed spacer and $L$. steelei $23 \mathrm{~S}-5 \mathrm{~S}$ rDNA internal transcribed spacer. Standard curves were generated using gDNA isolated from a known number of cells and considering one GE to one cell. Real-time PCR was carried out in $25 \mu \mathrm{l}$ reaction mixtures consisting of $1 \times$ SYBR Green PCR Master Mix (Applied Biosystems, Foster City, CA, USA), 300 nм of each forward and reverse primer and $5 \mu$ of the template DNA. Samples were amplified in an ABI Prism 7700 (Applied Biosystems) using the following thermal protocol: $10 \mathrm{~min}$ at $95^{\circ} \mathrm{C}$, followed by 40 cycles of $15 \mathrm{sec}$ at $95^{\circ} \mathrm{C}$ and $1 \mathrm{~min}$ at $60^{\circ} \mathrm{C}$. The specificity of each primer pair was confirmed by melting curve analysis and DNA sequencing.

\section{Immunoblot analysis of translocation of TEM-1 $\beta$-lactamase fusion proteins into amoebae}

Legionella strains expressing the TEM-1-LegC5 effector fusion protein were suspended in pond water containing $5 \mu \mathrm{g} \mathrm{ml}^{-1}$ chloramphenicol and $1 \mathrm{mM}$ Isopropyl $\beta$-D-1-thiogalactopyranoside and inoculated into amoebae cultures at MOI 50. After $5 \mathrm{~h}$ of incubation at $25^{\circ} \mathrm{C}$ (for $S$. palustris assays) or $30^{\circ} \mathrm{C}$ (for Acanthamoeba castellanii), amoebae trophozoites were harvested, washed with phosphatebuffered saline and lysed with 1\% NP-40. Cell lysates were centrifuged at $13000 \mathrm{~g}$ for $10 \mathrm{~min}$ at $4{ }^{\circ} \mathrm{C}$ to separate the soluble fraction, consisting of the amoeba cytosol and translocated effector proteins, from the insoluble fraction, containing the internalized bacteria. Samples from both fractions were subjected to sodium dodecyl sulfate polyacrylamide gel electrophoresis and immunoblotting, using antiTEM antibody. Proteins from sodium dodecyl sulfate-polyacrylamide gels were transferred onto nitrocellulose membranes (Millipore, Billerica, MA, USA) and blocked with $5 \%$ nonfat dried milk in trisbuffered saline with $0.1 \%$ Tween 20 (Sigma, St Louis, MO, USA). Membranes were then analyzed by western blot with monoclonal antibody directed to the TEM-1 $\beta$-lactamase as primary antibody and an anti-rabbit IgG peroxidase conjugate (1:1,000; Thermo Scientific, Waltham, MA, USA) as secondary antibody. Reactive bands on nitrocellulose membranes were revealed with the SuperSignal West Pico Chemiluminescent Substrate (Thermo Scientific). Image analyses were performed with Image Reader LAS-3000 (Fujifilm, Greenwood, SC, USA). 
Statistical analysis

Two-way ANOVA and Bonferroni's Multiple Comparison Tests (Prism 6.0; GraphPad Software) were used to compare differences in \% of active amoebae and \% of propidium iodide (PI)-positive S. palustris trophozoites incubated with Legionella strains. Two-way ANOVA and Bonferroni test were used to determine the earliest time point at which decline of $S$. palustris differed significantly from the control group in quantitative PCR (qPCR) assays.

\section{Nucleotide accession numbers}

The $18 \mathrm{~S}$ rRNA gene sequences of protists isolated in this study were deposited in the GeneBank database under accession numbers KF589954 (isolate CWPL), KF589955 (isolate MG33), KF607041 (isolate C37C6), KF607042 (isolate D5MG) and KJ561452 (isolate WPD37).

Supplementary Material are included as Supplementary Information.

\section{Results}

Impact of L. pneumophila on the structure of the eukaryotic microbial community in laboratory microcosms

In order to look for protists that consume $L$. pneumophila in different environments, we used laboratory microcosms (for details, see Materials and methods) to study the community structures of soil and water protists from a variety of sources. In order to examine the effect of $L$. pneumophila on the protist communities, we compared the effects of adding E. coli and L. pneumophila as potential sources of nutrients. In preliminary experiments, we found that when no bacteria were added to the microcosms, the endogenous nutrients were rapidly exhausted and many cysts appeared within $96 \mathrm{~h}$. Therefore, the addition of $E$. coli as a nutrient serves as a control for the specific effects of L. pneumophila in these experiments.

Several soil and fresh-water samples (Supplementary Table S3) were incubated in the presence of either $L$. pneumophila or E. coli in the microcosms. The analysis of the eukaryotic microbial community by $18 \mathrm{~S}$ SSU pyrosequencing revealed a substantial shift in the community structure between pre- and post-addition of Legionella to the microcosms and between the addition of $E$. coli or Legionella to the microcosms. Figure 1 shows the distribution of assigned OTUs among different groups of protists according to the commonly accepted higher-level classification of eukaryotes (Adl et al., 2005; 2012). OTUs assigned to representatives of the five super groups of eukaryotes (Amoebozoa, Opisthokonta, Excavata, SAR supergroup (Stramenopiles, Alveolates and Rhizaria) and Archeaplastida) were found in the samples analyzed in this study, suggesting that a diverse eukaryotic community was successfully established in each microcosm. Because our studies are focused on phagocytic free-living protists, OTUs affiliated to Fungi, Metazoa and Archeaplastida were discarded. We placed those eukaryotic taxa whose position is not yet established in the 'other Eukarya' group. OTUs with conflictive taxonomic assignments (when compared with different SSU databases) were placed in an 'unknown eukaryote' group.

In general, the addition of Legionella markedly decreased the abundance of Amoebozoa OTUs in the microcosms when compared with the same sample before the addition of bacteria (sample T0) or incubated with E. coli (sample Ec). Abundance of amoebae was higher before the addition of Legionella in 12 of the 14 experiments performed. All samples incubated with Legionella showed lower abundance of Amoebozoa OTUs than samples incubated with E. coli (sample Ec). Ciliates did not show a consistent pattern relative to the presence or absence of Legionella. Whereas Legionella resulted in lower richness of Amoebozoa, abundance of OTUs assigned to Cercozoa and Heterolobosea significantly increased in microcosms incubated with Legionella. Therefore, in absence of Legionella (samples T0 or EC), microcosms were dominated by OTUs affiliated to Ciliophora, Amoebozoa, Stramenopiles, Cercozoa or Euglenoza, depending on the nature of the environmental sample. In contrast, most of the microcosms incubated with Legionella were dominated by Heterolobosean and Cercozoan assigned OTUs. This shift in the structure of the community was observed for most of the microcosms, regardless of the nature of the environmental sample (Figure 1).

We focused our attention on those OTUs that flourished when Legionella was added to the microcosms (Table 1). Remarkably, some of them were present in more than two different environmental samples and consistently increased in abundance with Legionella. A statistical analysis was performed to determine whether the increase in abundance of those particular OTUs was associated with the addition of Legionella to the microcosms. As shown in Table 1, the increase in relative abundance of OTUs 434476 (Heterolobosea), 1284337 (Heterolobosea) and 53886 (Cercozoa, Paracercomonas sp.) was significantly $(P<0.05)$ associated with the presence of Legionella, suggesting that those protists were resistant to infection by $L$. pneumophila. Although other reasons might also explain these associations, we decided to test the hypothesis that specific protists able to graze on Legionella will flourish when this bacterium was added to the microcosm.

\section{Protists that resist L. pneumophila infection fall into} three different groups

Protozoan morphotypes able to grow in presence of L. pneumophila were isolated from microcosms incubated with Legionella (\#MG, \#WPL, \#WPD, 

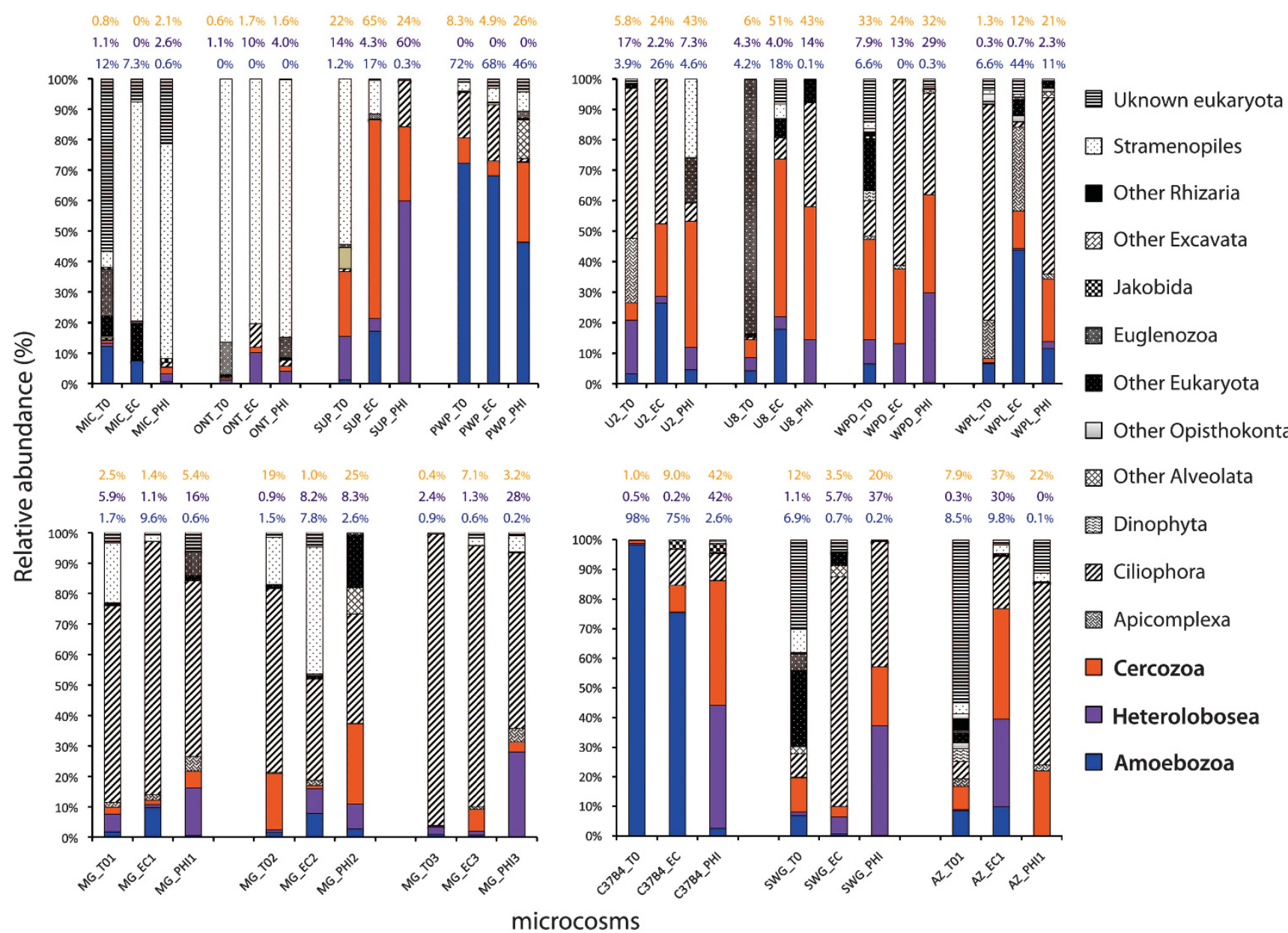

microcosms

Figure 1 Impact of L. pneumophila on the structure of eukaryotic microbial communities in laboratory microcosms. (a): Taxonomic affiliation of protist OTUs retrieved from different environmental samples incubated in the presence of E. coli (EC) or L. pneumophila str. Philadelphia-1 (PHI). Bars represent the relative abundance of protist taxa after rarefaction to correct for uneven sampling as described in the Materials and methods section. Sample identities are given on the $x$ axis. MIC: fresh-water sample from Lake Michigan. ONT: freshwater sample from Lake Ontario. SUP: fresh-water sample from Lake Superior. PWP: Pond-Washington Park (Chicago, IL, USA). U2: soil from Aguascalientes (Mexico). U8: soil from Neyaldi (India). WPD and WPR: soil samples from Washington Park (Chicago, IL, USA). MG: soil from Hyde Park (Chicago, IL, USA). C37B4: Core sample from ocean subsurface, Expedition IODP 311. SWG: Sewage (WWTP, Chicago, USA). AZ: soil from Tucson, AZ, USA. Sample-name_0: microcosm analyzed before the addition of E. coli or L. pneumophila. Sample-name_EC: microcosm incubated with $10^{7}$ cells of E. coli. Sample-name_PHI: microcosm incubated with $10^{7}$ cells of L. pneumophila Philadelphia 1. Relative abundance values (\%) of Cercozoa, Heterolobosea and Amoebozoa are highlighted in orange, purple and blue color, respectively. See Microcosm design in Materials and methods Section for details.

Table 1 OTUs found in more than one environmental sample that increased in abundance in presence of L. pneumophila

\begin{tabular}{|c|c|c|c|}
\hline OTU Id & Taxonomic affiliation & Samples & $\mathrm{P}$-value ${ }^{a}$ \\
\hline 1156079 & Amoebozoa; Flabellinea & PWP, U2 & N/A \\
\hline 434476 & Excavata; Heterolobosea; Vahlkampfiidae & SUP, SWG, U2, U8, MG & 0.0267 \\
\hline 1284337 & Excavata; Heterolobosea; Vahlkampfiidae; unclassified Naegleria & SWG, MG1, MG2, MG3 & 0.0277 \\
\hline 1249031 & Excavata; Heterolobosea; Vahlkampfiidae & ONT, WPM & N/A \\
\hline 1295870 & Excavata; Heterolobosea; unclassified heterolobosean & MIC, U8 & N/A \\
\hline 53886 & Rhizaria; Cercozoa; Cercomonanida; Cercomonadidae, Paracercomonas & $\begin{array}{l}\text { C37B4, MIC, ONT, SUP SWG, } \\
\text { PWP, U2, U8 WPL, MG1, MG2 }\end{array}$ & 0.0178 \\
\hline 291233 & Rhizaria; Cercozoa; soil Cercozoa & MG1, MG2 & N/A \\
\hline
\end{tabular}

Abbreviation: OTU, operational taxonomic unit.

${ }^{\text {a }}$ For those OTUs found in more than two environmental samples, a statistical test was performed to determine whether increase in abundance is associated with the presence of Legionella. $P$-value is the result of ANOVA test after Bonferroni test correction. Bold entries highlight those OTUs that are associated with the presence of Legionella in the microcosms according to the statistical analysis. 
\#PWP and \#AZ) and pure cultures were established as described in Materials and methods. Epifluorescence microscopy of mCherry-expressing Legionella was used to investigate the uptake and fate of the bacteria inside the cells. Using this approach, we were able to assign each protist to a different group based on its interactions with $L$. pneumophila (Figure 2). Group 1 includes amoebae (\#WPD37) that avoid uptake of L. pneumophila. Group 2 comprises ciliates and amoebae morphotypes (\#C37C6) that after ingestion release live Legionella packaged into pellets. Group 3 includes amoebae (S. palustris, \#WP11 and \#D5MG) and Cercozoan morphotypes (isolates \#CWPL and \#MG33) that consume L. pneumophila in digestive vacuoles. As shown in Figure 2, L. pneumophila is digested soon after phagocytosis by Group 3 organisms. The digestion of the bacteria imparted a diffuse red fluorescence on the digestive vacuoles, presumably caused by the release of mCherry from the lysed Legionella cells.

To determine whether these isolates are related to known protist species, we sequenced a $\sim 560 \mathrm{bp}$ region of their $18 \mathrm{~S}$ rDNA genes. The taxonomic affiliation of each isolate was determined by phylogenetic analysis and comparison with $18 \mathrm{~S}$ SSU sequences in the NCBI $\mathrm{nr}$ database. The resulting molecular genetic tree is presented in Supplementary Figure S1. The C37C6 $18 \mathrm{~S}$ sequence showed $100 \%$ identity to the $18 \mathrm{~S}$ rDNA of Acanthamoeba astronyxis. The $18 \mathrm{~S}$ sequence of CWPL had a $100 \%$ match to the $18 \mathrm{~S}$ rDNA of Paracercomonas sp. and Cercomonas sp. Isolate MG33 showed 97\% identity with unidentified
Cercozoan clones and Cercomonas sp. Isolate $S$. palustris was previously established as a novel genus and species by us (Anderson et al., 2011). The WPD37 $18 \mathrm{~S}$ sequence had $\leqslant 90 \%$ identity with $18 \mathrm{~S}$ rDNA of unidentified amoebozoa clones and its closest relative is Hartmannella sp. according to the phylogenetic analysis.

\section{S. palustris, Paracercomonas CWPL and Cercomonas} MG33 consume L. pneumophila as source of nutrition We used two approaches to demonstrate that the protists comprising Group 3 actually consume virulent Legionella as a source of food. First, TEM confirmed that L. pneumophila was consumed within digestive vacuoles of protozoan isolates $S$. palustris, Paracercomonas CWPL and Cercomonas MG33 (Figure 3). Second, we used real-time PCR to quantify GE of both protist trophozoites and Legionella, as a way to measure protozoan growth and L. pneumophila consumption. As shown in Figure 4, trophozoites of $S$. palustris, Paracercomonas CWPL and Cercomonas MG33 significantly $(P<0.01)$ increased in numbers by using $L$. pneumophila, strain JR32 as sole source of nutrition. Exponential increases in protozoan GE over time correlated with a 100- to 1000-fold decrease in JR32 $\mathrm{GE} \mu \mathrm{l}^{-1}$, indicating that protozoan growth resulted from consumption of JR32 cells (Figure 4).

\section{L. steelei kills S. palustris by a 'food-poisoning'} mechanism

Because some of the protozoan isolates are closely related to amoebae species known to support
Group 1
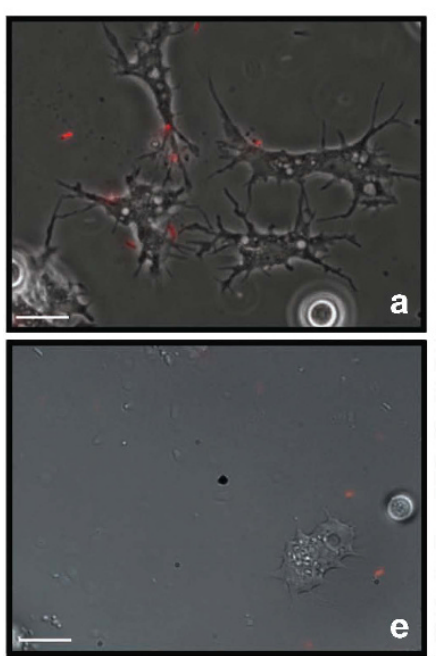

Group 2

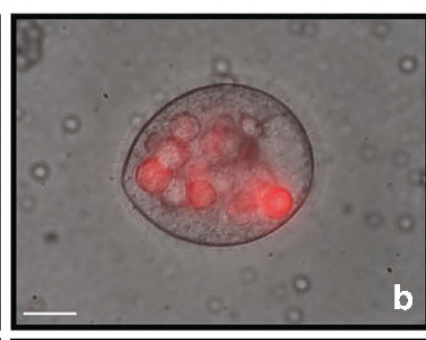

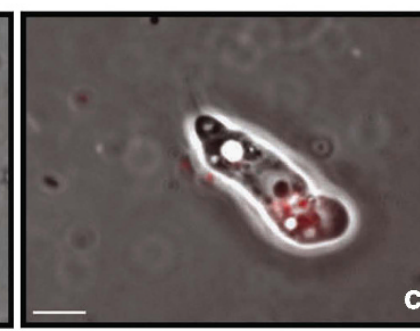

Group 3
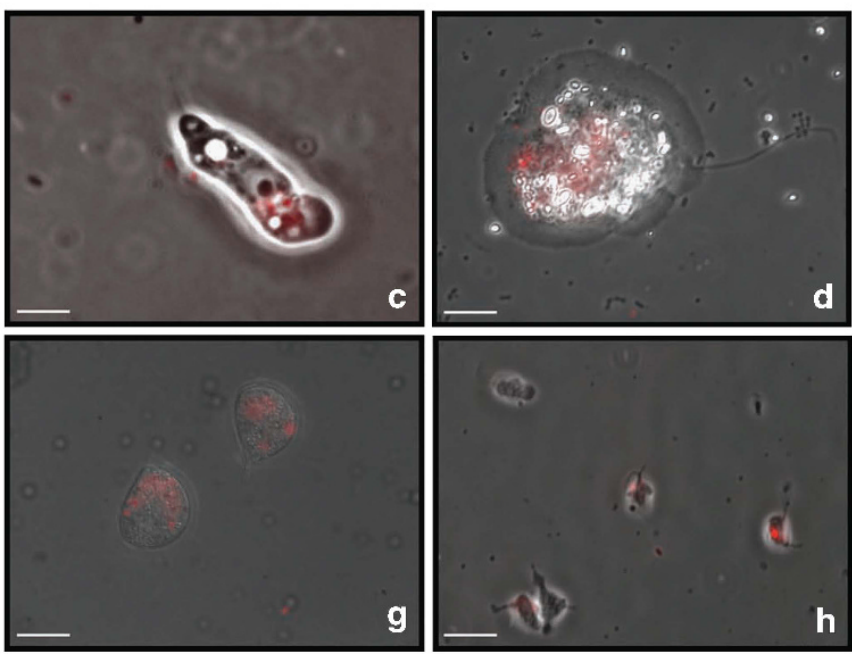

Figure 2 Protist morphotypes that resist L. pneumophila infection fall into three different groups. Combined DIC and epifluorescence images of protozoan isolates recovered from microcosms incubated with $L$. pneumophila Philadelphia-1. Pure cultures of each protist isolate (established as described in Materials and methods) were incubated with mCherry-expressing L. pneumophila at MOI of 1000. Uptake and survival of Legionella was followed from $2-72 \mathrm{~h}$ after the addition of the bacteria. Images showed correspond to $24 \mathrm{~h}$ time point. Similar results were observed for 2 and $48 \mathrm{~h}$. Group 1: protists that avoid taking up Legionella (pictures a and e). Group 2: protists that expel Legionella packaged into pellets (pictures b and f). Group 3: protists that consume Legionella. The digestion of the bacteria (pictures c, $\mathbf{d}, \mathbf{g}$ and $\mathbf{h}$ ) imparted a homogeneous red fluorescence on the digestive vacuoles, presumably caused by the release of mCherry from the lysed Legionella cells. Scale bar represents $10 \mu \mathrm{m}$. 

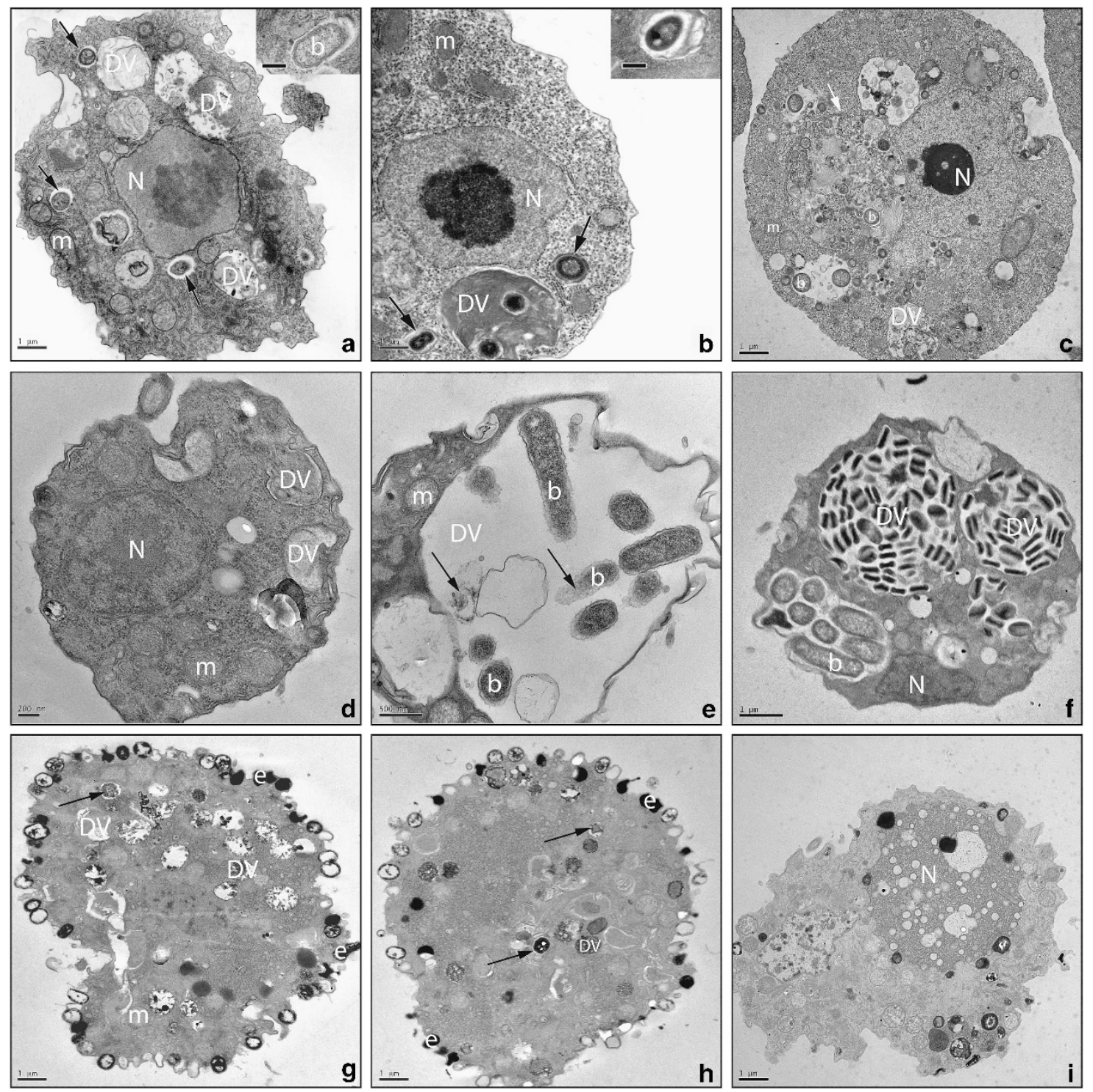

Figure 3 Protist grazers on L. pneumophila. Transmission electron micrographs of isolates S. palustris (a-c), Paracercomonas CWPL $(\mathbf{d}-\mathbf{f})$ and Cercomonas MG33 (g-i) showing control preparations with ingested E. coli (a, d, g) and those fed with L. pneumophila JR32 (b, e, h) or L. steelei IMVS3376 (c, f, i). TEM micrographs were taken $48 \mathrm{~h}$ after the addition of Legionella to the protist. b: bacteria; DV: digestive vacuole; e: extrusomes; m: mitochondria; N: nucleus. Black arrow indicates bacterial cell being digested. White arrow highlights an autophagosome. See Supplementary Figure S5 for more evidence of cellular damage in S. palustris trophozoites grazing on L. steelei.

L. pneumophila infection (Supplementary Figure S1), we wanted to find out if the protist isolates would consume other types of Legionella in the same way that they consume L. pneumophila, Serogroup 1. Therefore, we examined the interactions of different L. pneumophila strains and Legionella species (Supplementary Table S4) with isolates $S$. palustris, Paracercomonas CWPL and Cercomonas MG33. Co-cultures of each protist and Legionella strain were established in sterile pond water and monitored for protist growth and Legionella consumption for 10 days (data not shown). We observed that these protists were able to grow on different virulent $L$. pneumophila strains and other Legionella species but, surprisingly, the amoeba
S. palustris was killed by L. steelei strain IMVS3376. Quantification of $S$. palustris GE confirmed that the number of trophozoites significantly $(P<0.0001)$ decreased in the presence of L. steelei (Figure 4) in a dose-dependent manner (Supplementary Figure S2). In contrast, the $\operatorname{dot} A$ mutant of L. steelei was consumed and supported growth of this amoeba at all MOI used, as did heat-killed L. steelei cells (data not shown). These results suggest that living, wildtype L. steelei displays a cytotoxic response toward $S$. palustris that requires a functional Icm/Dot TFBSS secretion system and large numbers of bacteria. On the basis of these preliminary results, we focused our attention on the interactions between S. palustris and Legionella. 

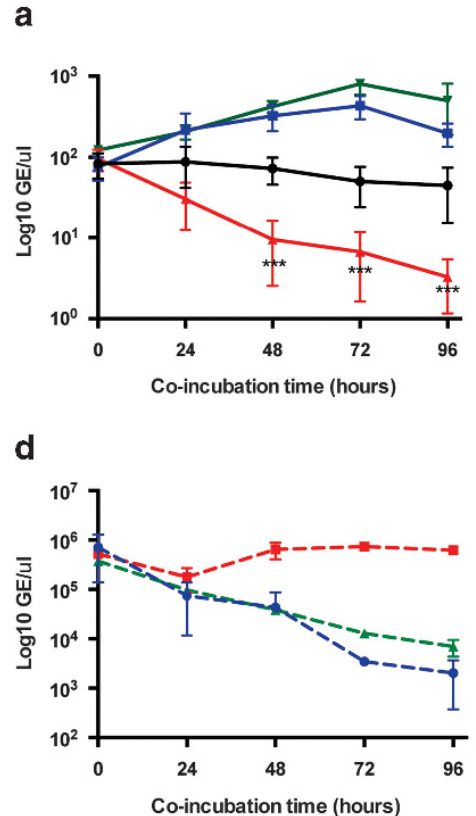

b

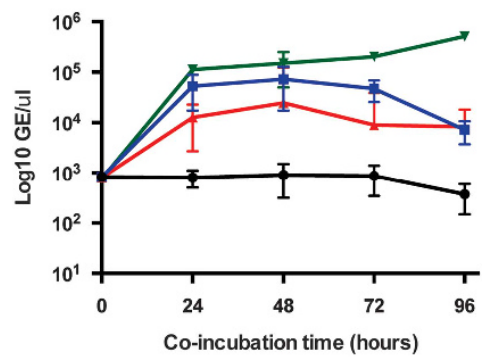

e

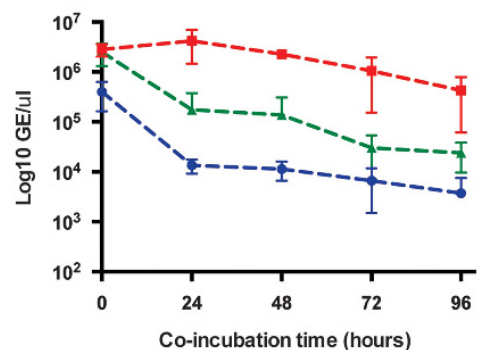

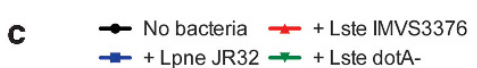

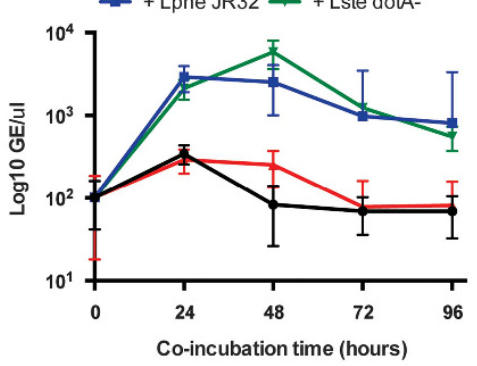

f

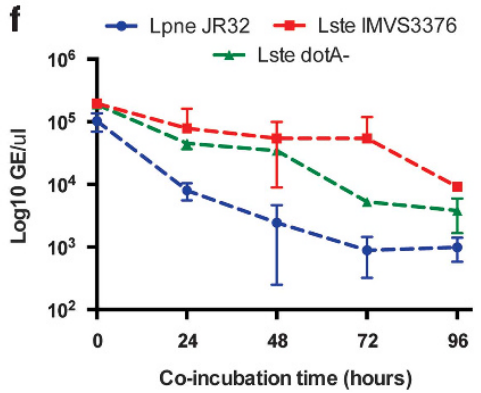

Figure 4 Growth of protozoan isolates S. palustris (a), Paracercomonas CWPL (b), Cercomonas MG33 (c) on L. pneumophila or L. steelei as food source. Protist growth (a-c) and Legionella consumption (d-f) was quantified by real-time PCR by targeting $18 \mathrm{~S}$ and $16 \mathrm{~S}$ rDNA genes, respectively. In total, $10^{4}$ trophozoites were cultivated in 96-well plates with $10^{7}$ cells of either L. pneumophila JR32 or L. steelei IMVS3376 wild-type or dotA- mutant in pond water at room temperature. On successive days, the number of Genome equivalents (GE) of protist trophozoites and Legionella was quantified by real-time PCR as described in Methods. Results shown correspond to the average of three independent experiments. Error bars indicate s.d. ${ }^{* * *}$ Indicates significant $(P<0.0001$, ANOVA and Bonferroni $)$ decline in the number of trophozoites when compared with the control group (no bacteria).

To further investigate whether L. steelei was able to kill S. palustris or inhibit its growth, we examined membrane integrity of the trophozoites by staining with PI, which is membrane impermeant and excluded from viable cells, but enters dead cells, binds to DNA and fluoresces. In co-cultures with wild-type $L$. pneumophila, only $0.2 \%$ of the trophozoites were PI positive. However, incubation with L. steelei led to extensive cell rounding (starting at $\sim 3 \mathrm{~h}$ after infection) and amoeba death; $55 \%$ and $70 \%$ of $S$. palustris trophozoites became permeable to PI after 72 or $96 \mathrm{~h}$ of incubation with L. steelei, respectively (Supplementary Figure S3). Similarly to qPCR data, the L. steelei $\operatorname{dot} A$ mutant strain did not result in staining by PI or cause cell rounding (Supplementary Figure S3), suggesting that the cytotoxic effect requires a functional Icm/Dot TFBSS secretion system. In order to distinguish whether the amoebae were simply encysting rather than dying in response to nutrient deprivation in the presence of overwhelming numbers of indigestible $L$. steelei, we performed a rescue experiment (Supplementary Figure S4). Removal of $L$. steelei cells after $24 \mathrm{~h}$ of co-culture rescued $50 \%$ of Solumitrus trophozoites. Actively grazing trophozoites were observed following the addition of $E$. coli cells as prey, indicating viable amoebae. Only $21 \%$ of amoebae could be rescued after $48 \mathrm{~h}$. In contrast, trophozoites could not be rescued after $72 \mathrm{~h}$ of incubation with $L$. steelei. Only $\sim 0.5 \%$ of the amoebae were able to grow on E. coli, indicating that most of the amoebae had not encysted but were dead by day 3 (Supplementary Figure S4).
As wild-type L. steelei grew robustly within and killed A. castellanii (Supplementary Figure S7), we investigated whether intracellular multiplication of L. steelei within $S$. palustris trophozoites is a cause of amoebal death. Co-cultures of Solumitrus with L. steelei were established and analyzed by qPCR and TEM (Figures 3-4). We found no evidence of L. steelei growing inside $S$. palustris. Real-time PCR quantification revealed that $L$. steelei GE did not significantly increase over time, indicating that the bacteria failed to multiply inside the amoeba (Figure 4). Moreover, replicative phagosomes were not observed in $S$. palustris trophozoites by TEM examination (Figure 3). Remarkably, at $48 \mathrm{~h}$ of coincubation, $S$. palustris trophozoites exhibited autophagosomes (Figure 3) and clear evidence of cell damage such as less granular cytoplasm (Supplementary Figure S5), damaged mitochondria and large vacuoles enclosing membranous whorls and granular masses of degraded cytoplasm (Supplementary Figures S5). In contrast, S. palustris grazing on L. pneumophila strain JR32 contained vacuoles where Legionella was consumed in a manner similar to digestive vacuoles containing E. coli prey (Figure 4). Cytotoxicity of wild-type L. steelei was dependent on the bacteria:protist ratio. Ratios lower than 100 were well tolerated and supported a discrete growth of the amoeba with negligible effects (Supplementary Figure S2). In contrast, ratios $\geqslant 500$ resulted in death of the amoebae, with trophozoites exhibiting a large number of autophagosomes. Although no cell 
damage was observed for Solumitrus grazing on the L. steelei $\operatorname{dot} A$ mutant, trophozoites also contained autophagosomes (Supplementary Figure S6), suggesting that the amoeba may use an autophagy-like mechanism to digest bacteria under conditions of a high ratio bacteria:amoeba. Autophagosomes were not observed in amoebae starving for $48 \mathrm{~h}$ or amoebae grazing on $L$. pneumophila, regardless of the bacteria:amoeba ratio, suggesting that this response was specific of the interaction between S. palustris and L. steelei.

Taken together, the qPCR data and microscopy observations confirm that ingested L. steelei cells were not able to productively infect $S$. palustris but were toxic to this amoeba. The fact that small amounts of L. steelei did not replicate intracellularly and were not toxic suggests that the bacteria killed $S$. palustris by a novel 'food-poisoning'-like mechanism that is not associated with intracellular multiplication.

L. pneumophila fails to translocate TFBSS effectors to S. palustris

One possible reason that $S$. palustris is able to predate L. pneumophila and L. steelei fails to grow is that the TFBSS may not be able to function properly in this context. In order to evaluate this possibility, we measured the ability of $L$. pneumophila and L. steelei to deliver effectors into $S$. palustris using a TEM-1, $\beta$-lactamase hybrid protein (Figure 5). $S$. palustris trophozoites were infected with wildtype strains of $L$. pneumophila or L. steelei that express TEM-1 ß-lactamase fused to the effector protein LegC5. The presence of the hybrid protein in the cytoplasm of the amoeba was detected by western blot. The presence of the TEM-LegC5 in the cytosol is based on its ability to be extracted from

a

\begin{tabular}{|c|c|c|c|c|c|}
\hline \multicolumn{6}{|c|}{ A. castellanii } \\
\hline \multicolumn{2}{|c|}{$\operatorname{dot} A-$} & \multicolumn{2}{|c|}{ JR32 } & \multicolumn{2}{|c|}{ Lste } \\
\hline$S$ & $P$ & $\mathrm{~S}$ & $P$ & $S$ & $\mathrm{P}$ \\
\hline & . & nimes & nomers & and & enes: \\
\hline
\end{tabular}

b

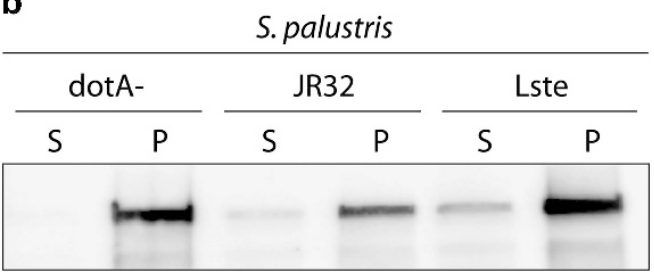

Figure 5 Translocation of hybrid protein TEM-1-LegC5 by wildtype L. pneumophila strain JR32; L. steelei and $\operatorname{dot} A$ mutant into A. castellanii (a) and S. palustris (b). Five hours after infection, amoebae were harvested and lysed. Cell lysates were centrifuged to separate the soluble fraction (S), consisting of the amoeba cytosol and translocated effector proteins; from the insoluble fraction (P), containing the internalized bacteria. Samples from both fractions were analyzed by western blot using anti-TEM-1 antibody. infected cells by the non-ionic detergent, NP-40. This detergent does not extract proteins from intact bacteria but does extract proteins that are present in the cytosol of amoebae. Translocation of TEM-LegC5 to $A$. castellanii following infection was measured as a positive control. Infection with $\operatorname{dot} A$ mutants expressing TEM-1-LegC5 was performed as a negative control for translocation. As shown in Figure 5, both Legionella species were able to translocate proteins into $A$. castellanii. In contrast, L. pneumophila failed to deliver the hybrid protein into $S$. palustris; whereas $L$. steleei was able to translocate only small amounts of the TEM-1- LegC5 protein. These data suggest that $L$. pneumophila is unable to translocate effectors into $S$. palustris via the Icm/Dot system. The fact that $L$. steelei translocates TEM-1-LegC5 to $S$. palustris inefficiently may explain why the bacteria cannot multiply intracellularly, and its killing of the amoebae requires a high MOI.

\section{Discussion}

The acquisition of virulence-related traits by pathogens with an environmental niche is poorly understood. It has been proposed that $L$. pneumophila may have acquired such traits as a consequence of selection for survival and replication in free-living protists (Molmeret et al., 2005). Thus, mechanisms that originally evolved to confer resistance to digestion by phagocytic protists may serve as virulence factors when Legionella accidentally infects human macrophages. As Rowbotham (1980) first reported the intracellular multiplication of $L$. pneumophila in Acanthamoeba spp. and Naegleria spp., numerous studies have shown that over 15 different species of free-living amoebae can serve as hosts for Legionella in vitro (Fields, 1996; Valster et al., 2010). In contrast, to our knowledge, the existence of protists that consume Legionella has never been investigated. This study provides the first experimental evidence of different environmental protist morphotypes that prey on virulent strains of L. pneumophila and other pathogenic species of the genus Legionella, supporting the hypothesis that predation by protozoa is an operative selective pressure for the acquisition and maintenance of virulencerelated traits in these bacteria.

The structure of the protist communities in the microcosms were determined both by the species composition in each sample and the ability of individual organisms to grow under the experimental conditions: nutrient availability and high concentration of L. pneumophila cells. Therefore, we expected that only protists resistant to Legionella would be recovered at the end of the experiment. The structure of each eukaryotic microbial community was analyzed by a RNA-based approach that allowed us to detect primarily metabolically active protists in the microcosms. DNA-based community analyses identify microorganisms in environmental samples regardless of their viability or metabolic 
activity (Stoeck et al., 2007). In contrast, as extracellular RNA is much less stable than DNA, the RNA-approach minimizes the problem of amplification from extracellular DNA or nonviable cells. Moreover, it has been shown that cellular rRNA content increases with growth rate and decreases with starvation (Stoeck et al., 2007).

The results in this study reveal new insights into the response of environmental protist communities to L. pneumophila. This species can exploit phagocytic protists as an environmental niche but depending on the host, the outcome of the Legionella-protozoa interaction may be different. In some cases, Legionella resists the digestion and kills the host, and in others, the protist digests the bacterium. In both soil and fresh-water samples, the structures of the protist communities were altered by L. pneumophila. The abundance of most Amoebozoa species was decreased, whereas the abundance of Cercozoan and Heterolobosean morphotypes that can consume Legionella as source of nutrition increased. Thus, the enrichment of these protists may result from the availability of an additional source of food (Legionella) that only these morphotypes are able to digest.

Heteroloboseans are amoebae, some with an alternate flagellate state, that are common in soil, freshwater and marine habitats. Two Heterolobosean species (Naegleria lovaniensis and Willaertia magna) have been reported to be partially resistant to infection by L. pneumophila by avoiding uptake of the bacteria (Declerck et al., 2005, Dey et al., 2009). According to the authors, only $2 \%$ of the trophozoites were infected after $72 \mathrm{~h}$. Two of the Legionella-consuming protists (D5MG isolated in this study) and S. palustris are indeed Heteroloboseans. Remarkably, they both are close relatives to the Heterolobosean Naegleria fowleri, which can serve as host for L. pneumophila (Newsome et al., 1985), indicating unknown specificity determinants in this prey-predator interaction. Cercozoa are globally the most abundant and genetically diverse predators in soil and include many of the most abundant and, therefore, ecologically significant soil, freshwater and marine protozoa (CavalierSmith and Oates, 2012). This group includes mostly amoeboid and flagellate protists. To date, no interactions between Legionella and Cercozoans have been reported. However, our data clearly show that these protists have an important role in the life cycle of Legionella as active predators. In accordance with our results, a study that aimed to detect new protozoan hosts for L. pneumophila showed high prevalence of an unidentified Cercozoan clone in samples from engineered water systems inoculated with Legionella (Valster et al., 2010).

The facile isolation of protists that graze on virulent strains of $L$. pneumophila provides unambiguous evidence of strong environmental selection for traits that protect Legionella from predation such as avoidance of phagosome-lysosome fusion. These traits are the same that confer virulence to Legionella in humans. As amoebae and macrophages have similar mechanisms of phagocytosis and killing of their prey (Davies et al., 1991), mechanisms to avoid digestion by amoebae or macrophages are also likely to be similar. We have unambiguously demonstrated that the amoeba $S$. palustris and the Cercozoans Paracercomonas CWPL and Cercomonas MG33 are able to consume virulent strains of $L$. pneumophila within a few hours post co-cultivation. The singular resistance of these protists to Legionella may be explained by factors relating to the bacterium or the protist. So far, the clearest growth defects of Legionella within amoebae have been shown for icm/dot mutants that are unable to evade phagosome-lysosome fusion (Segal and Shuman, 1999), or mutants that lack a large subset of effectors required for optimal growth in different hosts (O'Connor et al., 2011). Clearly, despite a functional TFBSS and complete repertoire of effectors, Legionella is unable to evade phagosome-lysosome fusion and survive within these protists. In fact, TEM images of ultrathin sections of $S$. palustris, Paracercomonas CWPL and Cercomonas MG33 grazing on L. pneumophila clearly showed that the bacteria were being rapidly degraded within phagosomes and did not establish a modified phagosome inside these protists. Furthermore, qPCR data demonstrated that these protozoans significantly decreased (2-3 log reduction) populations of L. pneumophila in co-culture.

All the L. pneumophila strains $(n=17)$ and Legionella spp. $(n=44)$ tested in this study were unable to multiply within $S$. palustris, Paracercomonas CWPL and Cercomonas MG33. However, we observed that Legionella isolates differed in their susceptibility to consumption by these protists, indicating unknown specificity determinants in these predator-prey interactions. In particular, L. steelei strain IMVS3376 killed the amoeba S. palustris by a food-poisoning-like mechanism; a cytotoxic response toward the protist that requires a high number of bacteria and a functional TFBSS.

Digestion of $L$. steelei by $S$. palustris involves the degradation of some bacteria in autophagosomes. Recent studies demonstrate selective autophagic degradation (xenophagy) of a variety of intracellular pathogens including bacteria, viruses and protozoan parasites (Knodler and Celli 2011; Levine et al., 2011). Antibacterial autophagy can capture not only cytosolic but also intravacuolar bacteria, by recognition of damaged phagosome membranes inflicted by bacterial activities (Knodler and Celli, 2011).

L. steelei was originally isolated from the respiratory tract of a patient in Australia (Edelstein et al., 2012). This Legionella species grows robustly in amoebae but fails to multiply within macrophages, although the bacterium is not killed (Supplementary Figure S7), suggesting that L. steelei can also evade phagosome-lysosome fusion in macrophages. Similarly, Edelstein et al. (2012) have previously shown 
that L. steelei failed to grow, but was not killed in A459 human lung cell lines. As this species does not grow axenically at $37^{\circ} \mathrm{C}$ (Edelstein et al., 2012), failure to multiply within human macrophages may be due to its restricted growth temperature. Though not an apparent human pathogen owing to its temperature growth restriction, L. steelei may represent an emerging human pathogen, as has been suggested for other amoebal pathogens (Lamoth and Greub, 2010).

Understanding how these protists graze on virulent Legionella, and subvert virulence-related traits, which promote intracellular growth in susceptible hosts, will provide important information about the roles of translocated effectors during infection as well as anti-virulence adaptations in protists that ensure continuity of remnant populations under selection by lethal pathogens such as Legionella spp. In addition, identification of the L. pneumophila genes that differ between successful and unsuccessful strains will lead to testable hypotheses for the mechanisms underlying their resistance to predation.

\section{Conflict of Interest}

The authors declare no conflicts of interest.

\section{Acknowledgements}

This work was supported by an award from NIAID, 5RO1AI23549 (H.A.S.) and funds from the Department of Microbiology, University of Chicago. F Amaro was supported by a postdoctoral fellowship from the Fulbright Commission and Ministry of Education of Spain. We thank our colleagues for supplying samples of various Legionella species, including Barry Fields and Claressa Lucas at CDC and Paul Edelstein at the University of Pennsylvania. We would like to thank the following individuals for supplying environmental samples used in this work: Dr Juan Carlos Gutiérrez from the Department of Microbiology III, Complutense University of Madrid, Dr Maureen Coleman from the Department of Geophysics at the University of Chicago and Dr Geeta K Rijal from the Metropolitan Water Reclamation District of Greater Chicago. The authors would like to express their appreciation to the Integrated Ocean Drilling Program shipboard technical, operations and engineering personnel of Integrated Ocean Drilling Program Expedition 311 for collecting, archiving and providing the ocean subsurface sample used in this research. Funding for Integrated Ocean Drilling Program was provided by the following agencies at the time of this expedition: European Consortium for Ocean Research Drilling, Ministry of Education, Culture, Sports, Science and Technology of Japan. Ministry of Science and Technology, People's Republic of China, National Science Foundation (United States). Any opinions, findings and conclusions or recommendations expressed in this publication are those of the author(s) and do not necessarily reflect the views of the participating agencies, Integrated Ocean Drilling Program Management International or the Integrated Ocean Drilling Program Implementing Organizations. This is Lamont-Doherty Contribution No. 7850.

\section{References}

Adl SM, Simpson AG, Farmer MA, Andersen RA, Anderson OR, Barta JR et al. (2005). The new higher level classification of eukaryotes with emphasis on the taxonomy of protists. J Eukaryot Microbiol 52: 399-451.

Adl SM, Simpson AG, Lane CE, Lukes J, Bass D, Bowser SS et al. (2012). The revised classification of eukaryotes. J Eukaryot Microbiol 59: 429-493.

Al-Quadan T, Price CT, Abu Kwaik Y. (2012). Exploitation of evolutionarily conserved amoeba and mammalian processes by Legionella. Trends Microbiol 20: 299-306.

Anderson OR, Wang W, Faucher SP, Bi K, Shuman HA. (2011). A new Heterolobosean amoeba Solumitrus palustris n. g., n. sp. isolated from freshwater marsh soil. J Eukaryot Microbiol 58: 60-67.

Bell T, Bonsall MB, Buckling A, Whiteley AS, Goodall T, Griffiths RI. (2010). Protists have divergent effects on bacterial diversity along a productivity gradient. Biol Lett 6: 639-642.

Berger KH, Isberg RR. (1993). Two distinct defects in intracellular growth complemented by a single genetic locus in Legionella pneumophila. Mol Microbiol 7: 7-19.

Caporaso JG, Lauber CL, Walters WA, Berg-Lyons D, Lozupone CA, Turnbaugh PJ et al. (2011). Global patterns of $16 \mathrm{~S}$ rRNA diversity at a depth of millions of sequences per sample. Proc Natl Acad Sci USA 108(Suppl 1): 4516-4522.

Cavalier-Smith T. (2002). The phagotrophic origin of eukaryotes and phylogenetic classification of Protozoa. Int J Syst Evol Microbiol 52: 297-354.

Cavalier-Smith T, Oates B. (2012). Ultrastructure of Allapsa vibrans and the body plan of Glissomonadida (Cercozoa). Protist 163: 165-187.

Davies B, Chattings LS, Edwards SW. (1991). Superoxide generation during phagocytosis by Acanthamoeba castellanii: similarities to the respiratory burst of immune phagocytes. Microbiology 137: 705-710.

Declerck P, Behets J, Delaedt Y, Margineanu A, Lammertyn E, Ollevier F. (2005). Impact of nonLegionella bacteria on the uptake and intracellular replication of Legionella pneumophila in Acanthamoeba castellanii and Naegleria lovaniensis. Microb Ecol 50: 536-549.

Dey R, Bodennec J, Mameri MO, Pernin P. (2009). Freeliving freshwater amoebae differ in their susceptibility to the pathogenic bacterium Legionella pneumophila. FEMS Microbiol Lett 290: 10-17.

Edelstein PH, Edelstein MA, Shephard LJ, Ward KW, Ratcliff RM. (2012). Legionella steelei sp. nov., isolated from human respiratory specimens in California, USA, and South Australia. Int J Syst Evol Microbiol 62: 1766-1771.

Edgar RC, Haas BJ, Clemente JC, Quince C, Knight R. (2011). UCHIME improves sensitivity and speed of chimera detection. Bioinformatics 27: 2194-2200.

Ensminger AW, Isberg RR. (2009). Legionella pneumophila Dot/Icm translocated substrates: a sum of parts. Curr Opin Microbiol 12: 67-73.

Fields BS. (1996). The molecular ecology of Legionellae. Trends Microbiol 4: 286-290.

Fouque E, Trouilhe MC, Thomas V, Hartemann P, Rodier MH, Hechard Y. (2012). Cellular, biochemical, and molecular changes during encystment of free-living amoebae. Eukaryotic Cell 11: 382-387. 
Franco IS, Shuman HA, Charpentier X. (2009). The perplexing functions and surprising origins of Legionella pneumophila type IV secretion effectors. Cell Microbiol 11: 1435-1443.

Fraser DW, Tsai TR, Orenstein W, Parkin WE, Beecham HJ, Sharrar RG et al. (1977). Legionnaires' disease: description of an epidemic of pneumonia. $N$ Engl J Med 297: 1189-1197.

Gao LY, Harb OS, Abu Kwaik Y. (1997). Utilization of similar mechanisms by Legionella pneumophila to parasitize two evolutionarily distant host cells, mammalian macrophages and protozoa. Infect Immun 65: $4738-4746$.

Gomez-Valero L, Rusniok C, Cazalet C, Buchrieser C. (2011). Comparative and functional genomics of Legionella identified eukaryotic like proteins as key players in host-pathogen interactions. Front Microbiol 2: 208.

Hahn MW, Hofle MG. (2001). Grazing of protozoa and its effect on populations of aquatic bacteria. FEMS Microbiol Eco 35: 113-121.

Jousset A. (2012). Ecological and evolutive implications of bacterial defences against predators. Environ Microbiol 14: 1830-1843.

Kilvington S, Price J. (1990). Survival of Legionella pneumophila within cysts of Acanthamoeba polyphaga following chlorine exposure. J Appl Bacteriol 68: 519-525.

Knodler LA, Celli J. (2011). Eating the strangers within: host control of intracellular bacteria via xenophagy. Cell Microbiol 13: 1319-1327.

Lamoth F, Greub G. (2010). Amoebal pathogens as emerging causal agents of pneumonia. FEMS Microbiol Rev 34: 260-280.

Luo ZQ. (2012). Legionella secreted effectors and innate immune responses. Cell Microbiol 14: 19-27.

Levine B, Mizushima N, Virgin HW. (2011). Autophagy in immunity and inflammation. Nature 469: 323-335.

Matz C, Kjelleberg S. (2005). Off the hook-how bacteria survive protozoan grazing. Trends Microbiol 13: 302-307.

McDade JE, Shepard CC, Fraser DW, Tsai TR, Redus MA, Dowdle WR. (1977). Legionnaires' disease: isolation of a bacterium and demonstration of its role in other respiratory disease. $N$ Engl J Med 297: 1197-1203.

Molmeret M, Horn M, Wagner M, Santic M, Abu Kwaik Y. (2005). Amoebae as training grounds for intracellular bacterial pathogens. Appl Environ Microbiol 71: 20-28.

Nash TW, Libby DM, Horwitz MA. (1984). Interaction between the legionnaires' disease bacterium (Legionella pneumophila) and human alveolar macrophages. Influence of antibody, lymphokines, and hydrocortisone. J Clin Invest 74: 771-782.

Nash TW, Libby DM, Horwitz MA. (1988). IFN-gammaactivated human alveolar macrophages inhibit the intracellular multiplication of Legionella pneumophila. J Immunol 140: 3978-3981.
Newsome AL, Baker RL, Miller RD, Arnold RR. (1985). Interactions between Naegleria fowleri and Legionella pneumophila. Infect Immun 50: 449-452.

Ninio S, Roy CR. (2007). Effector proteins translocated by Legionella pneumophila: strength in numbers. Trends Microbiol 15: 372-380.

O’Connor TJ, Adepoju Y, Boyd D, Isberg RR. (2011). Minimization of the Legionella pneumophila genome reveals chromosomal regions involved in host range expansion. Proc Natl Acad Sci USA 108: 14733-14740.

Page FC. (1988). A New Key to Freshwater and Soil Gymnamoebae With Instructions for Culture. Freshwater Biological Association: Cumbria.

Pernthaler J. (2005). Predation on prokaryotes in the water column and its ecological implications. Nat Rev Microbiol 3: 537-546.

Quast C, Pruesse E, Yilmaz P, Gerken J, Schweer T, Yarza P et al. (2013). The SILVA ribosomal RNA gene database project: improved data processing and web-based tools. Nucleic Acids Res 41: D590-D596.

Rowbotham TJ. (1980). Preliminary report on the pathogenicity of Legionella pneumophila for freshwater and soil amoebae. J Clin Pathol 33: 1179-1183.

Segal G, Purcell M, Shuman HA. (1998). Host cell killing and bacterial conjugation require overlapping sets of genes within a 22-kb region of the Legionella pneumophila genome. Proc Natl Acad Sci USA 95: 1669-1674.

Segal G, Shuman HA. (1999). Legionella pneumophila utilizes the same genes to multiply within Acanthamoeba castellanii and human macrophages. Infect Immun 67: 2117-2124.

Segal G. (2013). Identification of Legionella effectors using bioinformatic approaches. Methods Mol Biol 954: 595-602.

Shevchuk O, Jager J, Steinert M. (2011). Virulence properties of the Legionella pneumophila cell envelope. Front Microbiol 2: 74.

Solomon JM, Rupper A, Cardelli JA, Isberg RR. (2000). Intracellular growth of Legionella pneumophila in Dictyostelium discoideum, a system for genetic analysis of host-pathogen interactions. Infect Immun 68: 2939-2947.

Stoeck T, Zuendorf A, Breiner HW, Behnke A. (2007). A molecular approach to identify active microbes in environmental eukaryote clone libraries. Microb Ecol 53: 328-339.

Valster RM, Wullings BA, van der Kooij D. (2010). Detection of protozoan hosts for Legionella pneumophila in engineered water systems by using a biofilm batch test. Appl Environ Microb 76: 7144-7153.

Vogel JP, Andrews HL, Wong SK, Isberg RR. (1998). Conjugative transfer by the virulence system of Legionella pneumophila. Science 279: 873-876.

Supplementary Information accompanies this paper on The ISME Journal website (http://www.nature.com/ismej) 